This item was submitted to Loughborough's Research Repository by the author.

Items in Figshare are protected by copyright, with all rights reserved, unless otherwise indicated.

\title{
A comparative study on the effect of carbon fillers on electrical and thermal conductivity of a cyanate ester resin
}

PLEASE CITE THE PUBLISHED VERSION

https://doi.org/10.1016/j.polymertesting.2017.04.013

\section{PUBLISHER}

(C) Elsevier

VERSION

AM (Accepted Manuscript)

\section{PUBLISHER STATEMENT}

This work is made available according to the conditions of the Creative Commons Attribution-NonCommercialNoDerivatives 4.0 International (CC BY-NC-ND 4.0) licence. Full details of this licence are available at: https://creativecommons.org/licenses/by-nc-nd/4.0/

\section{LICENCE}

CC BY-NC-ND 4.0

\section{REPOSITORY RECORD}

Wang, Xiao, Yuntao Zhao, Jie Jin, and Mo Song. 2017. "A Comparative Study on the Effect of Carbon Fillers on Electrical and Thermal Conductivity of a Cyanate Ester Resin”. figshare. https://hdl.handle.net/2134/24916. 


\title{
A comparative study on the effect of carbon fillers on electrical and thermal conductivity of a cyanate ester resin
}

\author{
Xiao Wang a, *, Y untao Zhao a, Jie Jin b, Mo Song b \\ a Chongqing Institute of Green and Intelligent Technology, Chinese Academy of Sciences, \\ Chongqing 400714, China \\ b Department of Materials, Loughborough University, Loughborough, Leicestershire LE11 3TU, \\ United Kingdom
}

\begin{abstract}
Carbon fillers including multi-walled carbon nanotubes (MWCNTs), carbon black (CB) and graphite were introduced in a cyanate ester (CE) resin, respectively. The effects of the fillers on the electrical and thermal conductivity of the resin were measured and analyzed based on the microscopic observations. MWCNTs, CB and graphite exhibited percolation threshold at $0.1 \mathrm{wt} \%, 0.5 \mathrm{wt} \%$ and $10 \mathrm{wt} \%$, respectively. The maximal electrical conductivity of the composites was $1.08 \mathrm{~S} / \mathrm{cm}, \quad 9.94$ $10-3 \mathrm{~S} / \mathrm{cm}$ and $1.70 \quad 10 \longrightarrow 5 \mathrm{~s} / \mathrm{cm}$.
\end{abstract}

MWCNTs showed the best enhancement on the electrical conductivity. The thermal behavior of the composites was analyzed by calorimetry method. Incorporation of MWCNTs, CB and graphite increased the thermal conductivity of CE resin by $90 \%, 15 \%$ and $92 \%$, respectively. Theoretical models were introduced to correlate the thermal conductivity of the CE/MWCNTs composite. The interfacial thermal resistance between CE resin and MWCNTs was $8 \quad 10-8 \mathrm{~m} 2 \mathrm{~K} / \mathrm{W}$ and the straightness ratio was 0.2 . The MWCNTs were seriously entangled and agglomerated. Simulation results revealed that thermal conductivity of the CE/MWCNTs composites can be substantially elevated by increasing the straightness ratio and/or filler content of MWCNTs.

\section{Introduction}

Development of polymer composites with carbon fillers has become an attractive topic in tailoring the electrical and thermal properties of the insulating or low conductive polymer matrix [1e6]. Compared with traditional fillers, carbon fillers possess high surface to volume ratio and aspect ratio. They are significantly effective for enhancing the performance of polymer at low incorporation, owing to their superior conductivity, unique structure, excellent physicochemical stability and thermal stability [7e11].

However, improvements on the dispersion of carbon fillers and their interactions with polymers are the main challenges that limit the performance promotion of the composites [12e14]. Optimizing network structure and distribution of fillers is necessary to form a conductive path in matrix [14e17].

Recently, there is an increasing demand for high-performance thermosetting resins in industry [18]. The thermosets/carbon fillers composites show good performance as anti-static materials, conductive coating, electric heating devices, bipolar plate, etc. [17,19e21]. In order to extend their applications, thermosets/carbon fillers composites have been developed with improved electrical and thermal properties. Florian et al. [8] prepared epoxy composites by adding CNTs and CB. The percolation threshold was observed at low filler concentrations. The thermal conductivity was slightly increased. Similar result was obtained by investigating the conductivity of epoxy/MWCNTs composites in Vahedi's [22] research. An et al. [19] investigated the electric heating behavior of epoxy/graphene composites. The electrical resistance of composites varied dramatically with increasing the graphene content.

The composite showed rapid temperature response and good stability. Effects of graphite on the properties of epoxy resins were also studied. However, the improvement of thermal conductivity was 
always much smaller than the corresponding improvement in mechanical properties [23,24]. The increase in the electrical conductivity was not notable [25].

Cyanate ester resin is one of the most important high performance thermosets that provide excellent mechanical properties inherent flame retardancy, excellent mechanical strength, and good chemical resistance [26]. CE resins have been widely utilized in aerospace and defense industries. Compared with other thermosets, CE resins are more attractive with low moisture absorption, surface finish and dimensional stability [27e30]. The glass transition temperature of CE resin used in this study is over 400

C. CE resins have been widely proposed as replacements for epoxy resins in high temperature applications [31]. However, the electrical and thermal properties of CE resins are not satisfactory. The investigations on these properties of $C E$ resins are inadequate $[32,33]$. The low thermal conductivity of CE resins induces considerable accumulations of thermal energy, which could affect its mechanical properties for long term usage at elevated temperature. Therefore, the improvement on the thermal conductivity becomes quite important. For a successfully enhanced CE resin composite, an effective conductive network is essential. It can be actualized based on a good dispersion of the carbon fillers in matrix, but the dispersion has been challenging due to the high viscosity and high curing temperature of CE resins. Moreover, each type of carbon filler has its unique structure including dimension and aspect ratio. The effects of the filler structure on the electrical and thermal properties of CE resins are still unclear. In our previous work $[27,34]$, a CE resin, PT-30, composites were prepared by incorporation of carbon fillers. The processing and curing dynamics of the composites were studied. The dispersion and network formation of the fillers in CE matrix were sufficiently discussed. Based on this knowledge, in this study, the influences of different types of carbon fillers on the electrical and thermal conductivity of PT-30 are analyzed. We attempt to compare the enhancement effects of these fillers, and suggest appropriate applications of the CE resin composites, in order to guide the further development of the CE composites.

\section{Experimental}

\subsection{Materials}

Cyanate ester resin, Primaset PT-30 was purchased from LONZA Ltd. The molecular structure of CE resin is shown in Fig. 1. Ultrafine carbon black powder with particle size about $40 \mathrm{~nm}$ was supplied by Cobalt, Belgium. Purified multi-walled carbon nanotubes (MWCNTs) were purchased from Chengdu Institute of Organic Chemistry, Chinese Academy of Science. The MWCNTs (purity > 95\%) had a length of approximately $50 \mathrm{~mm}$ and diameter of $8 \mathrm{e} 15 \mathrm{~nm}$. The density was about 2.1 $\mathrm{g} / \mathrm{cm}$ 3. Ultrafine grade graphite with particle size $4 \mathrm{e} 7 \mathrm{~mm}$ was received from Qingdao Kropfmühl Graphite Company, China.

2.2. Preparation of PT-30 composites. The carbon fillers MWCNTs, CB and graphite were fully dried and dispersed in the PT-30 matrix by strong mechanical stirring for $2 \mathrm{~h}$ at 100

C, respectively. The stirring speed was $1000 \mathrm{rpm}$. The composites were produced at the same processing conditions, by varying filler content up to $10 \mathrm{wt} \%$ for each type of carbon filler. The mixtures were then placed in a vacuum oven ( $800 \mathrm{mbar}$ ) for 1 day to remove residual moisture. The mixtures was poured into a mould and cured for 2 h at 220

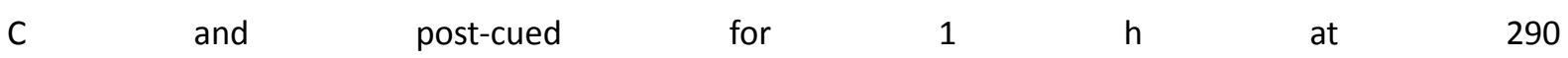


C.

\subsection{Characterization}

A Field-Emission Gun Scanning Electron Microscopy (FEGSEM) (LEO 1530VP instrument) was used to observe the morphology of fracture surface of the composites. Before the observation, the samples were freeze-fractured in liquid nitrogen and coated with gold by a sputter coater for 60s. Electrical conductivity of composites was measured using a Keithley Semiconductor Characterization System 4200-SCS. A direct voltage, V, was loaded on the specimen, and the current, I, was obtained. The electrical conductivity, s, can be calculated by the following equation:

$\sigma=\mathrm{L} / \mathrm{S} \times \mathrm{I} / \mathrm{V}$

where $L$ is the length of the cuboid sample, $S$ is the cross-sectional area. Before measurements, Silver paste was deposited on the top and bottom sides of each specimen, in order to get steady values when they were contacted with the probes. At least three specimens were tested for the electrical conductivity to obtain an average value for each composite. A standard model of TA instruments differential scanning calorimetry (DSC) 2920 Calorimetry was utilized to evaluate the thermal conductivity [35]. Fig. 2 illustrated the setup of DSC test for thermal conductivity. Pure indium (melting temperature: 156.7

\begin{tabular}{|c|c|c|c|c|c|c|c|c|c|c|c|}
\hline C) & was & the & calibration & substance. & The & tests & were & from & 100 & to & 180 \\
\hline $\mathrm{C}$ & & at & & a & heating & & rate & & of & & 10 \\
\hline
\end{tabular}

C min-1. The thermal conductivity value was obtained from calculation based on the following equation:

$\lambda=H / S \times \phi / \Delta T$

where $\lambda$ is the thermal conductivity, $H$ is the height of the sample, $S$ is the cross-sectional area, and $\phi / \Delta T$ is the slope of the melting curve of indium, which was obtained from the DSC plot. At least three specimens were tested for the thermal conductivity to obtain an average value for each composite.

\section{Results and discussion}

The incorporation of carbon fillers into the PT-30 induced an electrical conduction, by forming a conductive pathway. The lowest filler concentration, which is known as percolation threshold, is necessary to achieve conductivity, s 10 $-8 \mathrm{~s} / \mathrm{cm}$. The percolation theory states the critical amount of fillers required for the transition of composites from insulating to conductive. The electrical conductivity of the PT-30 composites was measured and plotted in Fig. 3 . It can be found that the percolation threshold of PT-30/ MWCNTs composite occurred at very low filler content, which was $0.1 \mathrm{wt} \%$. The MWCNTs showed excellent improvement on the electrical conductivity of PT-30, owing to their high aspect ratio. In contrast, $2 \mathrm{wt} \% \mathrm{CB}$ was required to achieve a similar conductivity. The percolation threshold of PT-30/CB composite was $0.5 \mathrm{wt} \%$. 


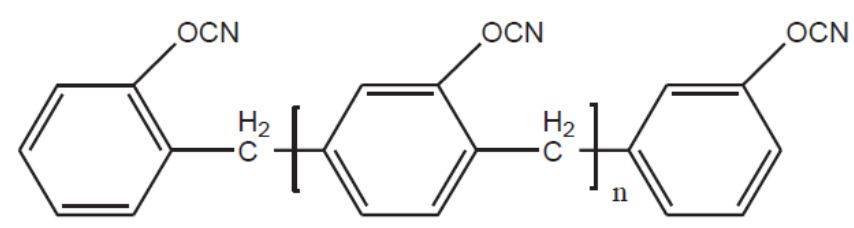

PT-30

Fig. 1. Molecular structure of PT-30 resin.

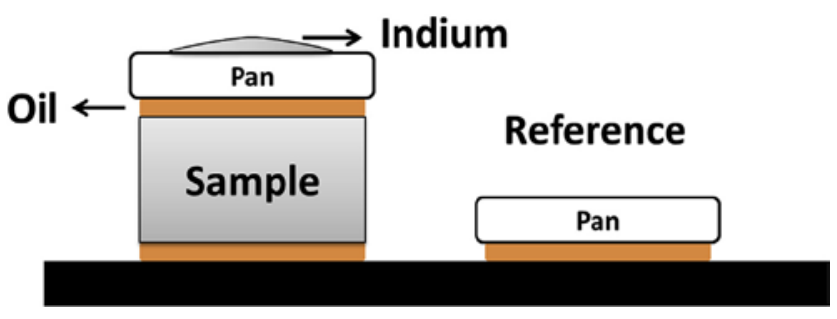

Fig. 2. The setup of DSC test for thermal conductivity

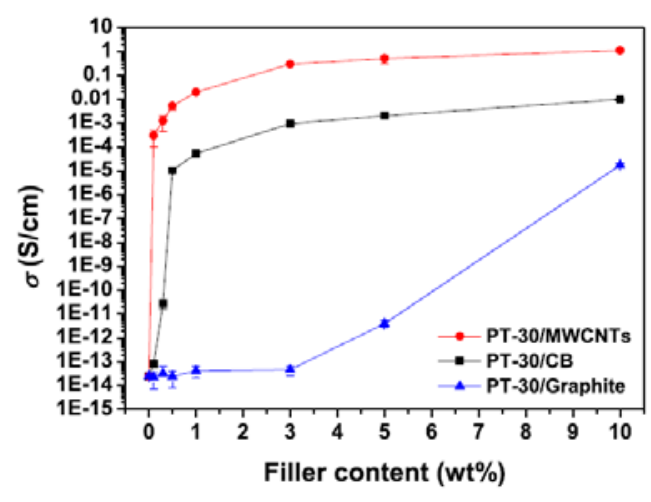

Fig. 3. Electrical conductivity of PT-30 composites with MWCNTs, CB and graphite at various contents

Therefore, the fiber shaped fillers showed a lower percolation threshold than that of spherical fillers in the PT-30 resins.MWCNTs and CB further enhanced the electrical conductivity of the PT-30 by increasing the filler content. A significant improvement can be observed at about 1 wt\% filler, and the conductivity became steady after $3 \mathrm{wt} \%$. The SEM morphology of the PT-30 composites with $1 \mathrm{wt} \%$ MWCNTs and $1 \mathrm{wt} \%$ CB was presented in Fig. 4 (a) and (b), respectively. A good dispersion of MWCNTs in the matrix was achieved, which induced an effective network for conduction. Hence, the improvement on the conductivity was substantial. For the PT-30/CB composite, it can be observed that the CB particles slightly agglomerated. However, the slight agglomeration did not significantly affect the conductivity enhancement, as the effective path for conduction was built up. The arrangement of CB particles and agglomerates could act as conducing backbone. The conduction behavior could be mainly described by two mechanisms. Firstly, the intra-agglomerate transportation of electrons occurred between the physically contacted CB particles. Secondly, for the interagglomerate or inter-particle conduction, where actual physical contact of neighboring fillers was not presented, the conduction took place via tunneling 
between the discontinuous conducting phases. Percolation occurred at low concentration. Further increasing filler content could result in the decrease of inter-filler gaps, which assisted the tunneling of electrons. Thus, the electrical conductivity continuously increased, although the dispersion quality may decrease as the increase of $\mathrm{CB}$ loadings. On the other hand, graphite has a higher aspect ratio than $\mathrm{CB}$. It would theoretically lead to a good electrical conduction in the resin matrix.

However, the graphite showed a much higher percolation threshold of $10 \mathrm{wt} \%$, compared with that of MWCNTs and CB. As shown in Fig. 4 (c), the average particle to particle distancewas over $10 \mathrm{~mm}$. The increased inter-particle gaps made the tunneling of electrons hard between particles. Thus, higher filler content was required to form a percolated network for PT-30/graphite composite. The incorporations of carbon fillers showed significantly different effects on the electrical properties of the PT-30 resins. MWCNTs exhibited excellent electrical conductivity at low concentration. It enabled PT-30/MWCNTs wider utilizations such as electromagnetic interference (EMI) shielding applications, whose required minimal electrical conductivity was $10-1 \mathrm{~S} / \mathrm{cm}$. For the PT- 30/CB composites with intermediate conductivity, they could be developed as anti-static coatings with $\mathrm{s}$ of at least 10 $8 \mathrm{~S} / \mathrm{cm}$.

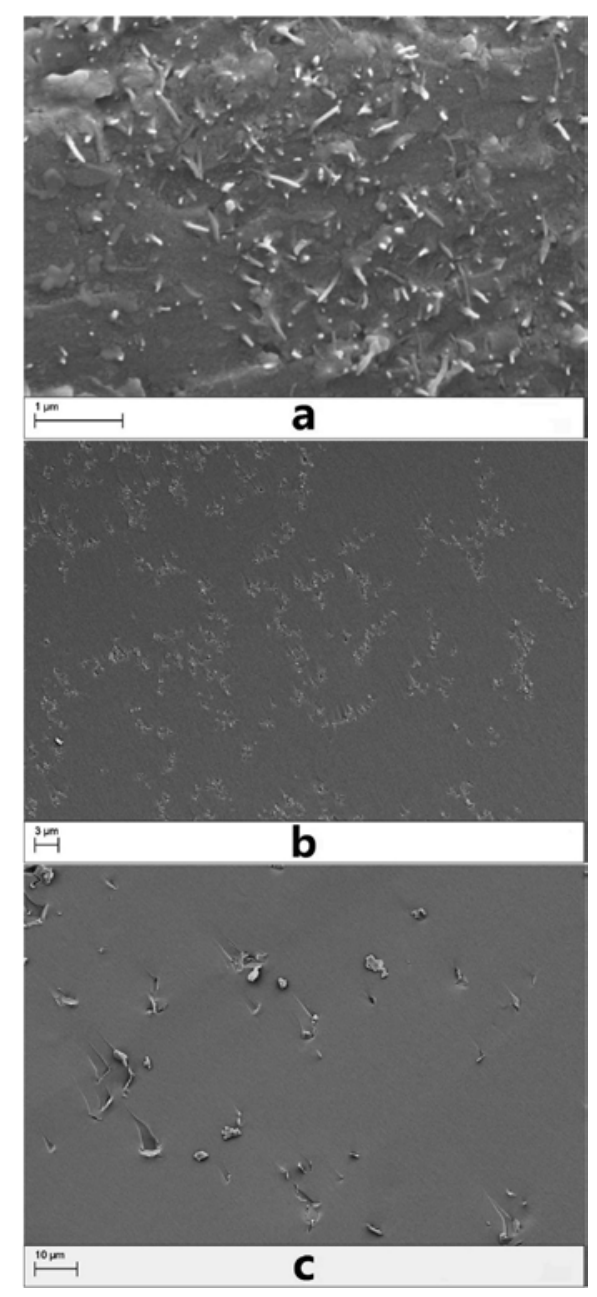

Fig. 4. SEM images of PT-30 composite containing 1 wt\% (a) MWCNTs, (b) CB and (c) graphite.

The formation of percolated network is essential for the conduction of electrons in composites. With regard to thermal conductivity, however, it shows no direct correlation with the percolation mechanism 
[8]. In contrast, the transport of thermal energy in PT-30/carbon filler composites is related to a phonon conduction mechanism, where scattering effects dominates. In general, carbon nanofillers with one-dimension or two dimension structures are ideally for the enhancement of thermal conduction, according to their crystalline graphite structure and high aspect ratio. They show much higher thermal conductivity than of the amorphous PT-30 matrix, owing to their high aspect ratio. The phonon transport can be perfectly occurred through a high free length of path with a high number of phonon vibrational models been activated in the crystalline lattice structure. However, scattering effects, which mainly occur in interfacial boundary between particle and matrix, significantly influence the phonon transport and thus the efficiency of thermal conduction in the PT-30 composites. The level of the scattering is proportional to the overall interface size and the number of defects.

Fig. 5 shows the thermal conductivity of PT-30 composites with different carbon fillers. As expected, the addition of carbon black shows a gentle improvement on the thermal conductivity. The low aspect ratio of $\mathrm{CB}$ allowed the conduction of phonons over short distance but with massive transitions from particle to particle, which was adverse for thermal conduction. Also, the high specific surface area of CB gave rise to a tremendous contact surface area that acted as interface in PT-30. The coupling losses of the phonon conduction at the interface were permanent. The scattering was significant. In contrast to CB, MWCNTs had much smaller specific contact area but higher aspect ratio, which enabled a higher free length of conductive paths. Besides, the internal layers of MWCNTs did not contact with the PT-30 matrix, shielding the conducted phonons. It resulted in a minor coupling losses, thus MWCNTs showed a better enhancement of thermal conductivity, compared with $\mathrm{CB}$. Furthermore, the incorporation of graphite showed a comparable improvement on the thermal conductivity as MWCNTs. The internal graphene layers were shielded from coupling loss, which contributed to the thermal conductivity elevation. Therefore, considering the high electrical percolation threshold at $10 \mathrm{wt} \%$, PT-30/graphite composites can be developed as electrical insulating devices that require enhanced thermal conductivity.

It should be also noticed that MWCNTs exhibited the best enhancement of thermal conductivity at filler loadings of up to $3 \mathrm{wt} \%$, among the carbon fillers. However, the conductivity was lower than that of PT-30/graphite composites at higher filler concentrations. The enhancement effect of MWCNTs became less effective. It is conceivable that massive CNT junctions were produced as the increasing amount of MWCNTs in PT-30 matrix, owing to the agglomeration and entanglement of CNTs. These junctions acted as defects that reduced the thermal conductivity. The transition of phonons from nanotube to nanotube occurred by direct coupling in the junctions. It induced an intense phonon boundary scattering and decreased the thermal conductivity. Therefore, it is important to further investigate the thermal conductivity of the PT-30/ MWCNTs composite, relating to the arrangement, agglomerate state and interfacial thermal resistance of theMWCNTs in the PT-30 matrix. The thermal conductivity of PT-30/MWCNTs composites was fitted by theoretical models to understand the conductive mechanism. The simple mixing model (Eq. (3)) and parallel electric circuit model (Eq. (4)) were firstly introduced, 


$$
\begin{aligned}
& \frac{k_{c}}{k_{m}}=v_{m}+\frac{k_{f} v_{f}}{k_{m}} \\
& \frac{k_{c}}{k_{m}}=\frac{1 / k_{m}}{v_{m} / k_{m}+v_{f} / k_{f}}
\end{aligned}
$$

where $\mathrm{kc}, \mathrm{km}$ and $\mathrm{kf}$ are the thermal conductivity of composite, matrix and filler, respectively. $\mathrm{nm}$ and $\mathrm{nf}$ denote the volume fraction of matrix and filler, respectively. The correlations of the two models with experimental results are shown in Fig. 6 . The first model was developed based on the formation of an internal network, where interactions between filler and matrix phases were neglected. This model maximized the effect of the MWCNTs phase, and resulted in an overestimated thermal conductivity of composite. Hence, it defined an upper bound for the thermal conductivity. The second model assumed that the fillers were isolated in the matrix, and the composite was homogeneous. It should be noted that the fillers can be described as isolated phase only at its volume fractions below percolation threshold. In the PT-30/MWCNTs system, this model was inappropriate and could be regarded as lower bound. Both of the two models did not realistically describe the thermal conductivity of the composite. An inclusion model [36] was introduced to simulate the experimental results of MWCNTs in PT-30 matrix. For the PT-30 composites with low incorporations of randomly oriented straight MWCNTs, the thermal conductivity of composite was calculated from the following equations:

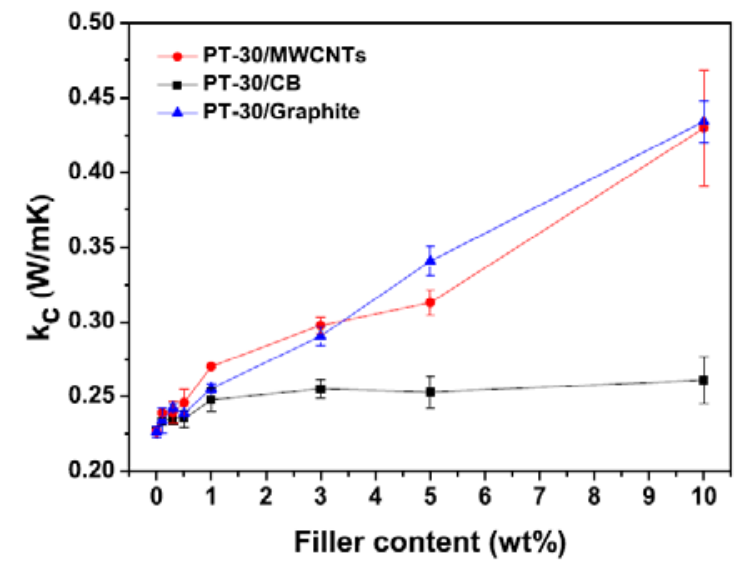

Fig. 5. Thermal conductivity of PT- 30 composites with MWCNTs, CB and graphite at various contents.

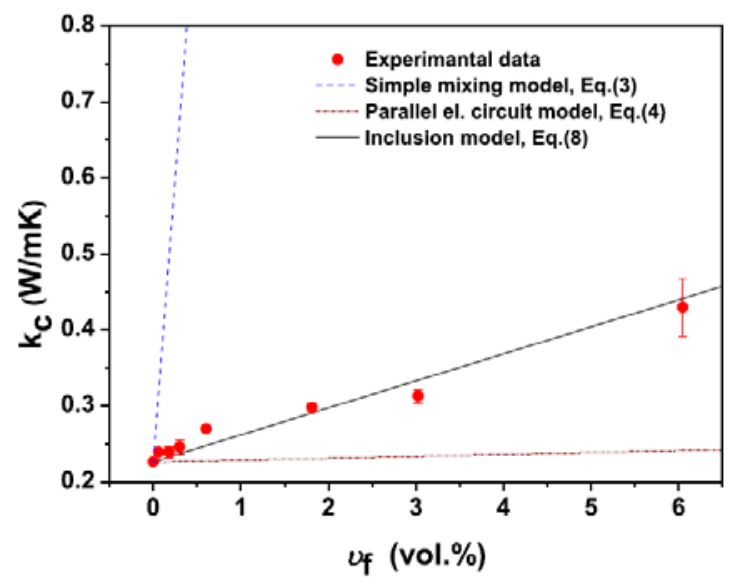

Fig. 6. Correlation of experimental results for PT-30/MWCNTs composites with theoretical models. 


$$
\begin{aligned}
\frac{k_{c}}{k_{m}}=1 & +\frac{v_{f}}{3}\left[\frac{1}{\left(k_{33}^{f} / k_{m}-1\right)^{-1}+H}\right. \\
& \left.+\frac{2}{\left(k_{11}^{f} / k_{m}-1\right)^{-1}+(1-H) / 2}\right] \\
H(\beta)= & \frac{1}{\beta^{2}-1}\left[\frac{\beta}{\beta^{2}-1} \operatorname{In}\left(\beta+\sqrt{\beta^{2}-1}\right)-1\right]
\end{aligned}
$$

where $\beta$ is the aspect ratio ( $\beta \frac{1}{4} \mathrm{~L} / \mathrm{D}$ ) of the MWCNTs ( $L 1 / 4$ average length; $D$ 1 $1 / 4$ diameter), $k^{f} 33$ and $k^{f} 11$ are the longitude and transverse thermal conductivities of the MWCNTs, respectively. In our study, $\mathrm{km} 1 / 4$ $0.2263 \mathrm{~W} / \mathrm{mK}$, kf $\quad 1 / 4 \quad 2000 \quad \mathrm{~W} / \mathrm{mK}$ and $\beta \quad 1 / 4 \quad 4000$. Thus $\mathrm{kf} / \mathrm{km}[1$ and $\mathrm{H} \quad 1 / 4 \quad 5$ 10_7. In this case, the calculated second term in the square bracket in Eq. (5) was four orders smaller than the first term. Therefore, the second term was negligible and Eq. (5) can be simply expressed as following:

$$
\frac{k_{c}}{k_{m}}=1+\frac{v_{f} / 3}{k_{m} / k_{33}^{f}+H}
$$

In practice, however, the MWCNTs were non-straightly distributed in PT-30 matrix according to the SEM observation. Also, interfacial thermal contact resistance, which was known as Kaptiza resistance [37], Rk, should be taken into account. In order to define the non-straightness of the individual MWCNTs in PT-30, average end to end distance, Le, is introduced to show the equivalent conductive length of MWCNTs, and $d 1 / 4 L e=L$ is the straightness ratio. Based on the above analysis, a refined equation can be achieved.

$$
\frac{k_{c}}{k_{m}}=1+\frac{\delta v_{f} / 3}{k_{m} /\left[\delta k_{33}^{f} /\left(1+2 R_{k} k_{33}^{f} / L\right)\right]+H(\delta \beta)}
$$

As shown in Fig. 6, a good agreement can be observed between the experimental results and the model based on Eq. (8). The parameters of MWCNTs states in CE matrix from the modeling were listed in Table 1. The fitted $d 1 / 40.2$ and Rk $1 / 48 \quad 10-8 \mathrm{~m} 2 \mathrm{~K} / \mathrm{W}$. The average end to end distance was Le $1 / 4 \mathrm{dL} 1 / 410 \mathrm{~mm}$. The effective aspect ratio, be $1 / 4 \mathrm{db} 1 / 4800$. Compared with the raw aspect ratio $b 1 / 44000$, it can be concluded that the MWCNTs were seriously entangled and agglomerated. The potential of the MWCNTs enhancement was not fully explored. The difference between the maximum and the fitted values resulted from agglomerations of MWCNTs in PT-30 resins and possible shortening of the length that may occur during processing. Fig. 7 shows the thermal conductivity of PT-30/MWCNTs composites as a function of volume fraction of MWCNTs at variable straightness ratio. It indicates that the increase of $d$ and/or filler content substantially elevate the thermal conductivity of the PT-30/MWCNTs 
composites.

Table 1

Inclusion modeling of MWCNTs states in PT-30 matrix.

\begin{tabular}{llllll}
\hline Parameters $^{\mathrm{a}}$ & $\beta$ & $\beta_{e}$ & $\delta$ & $L_{e}(\mu \mathrm{m})$ & $R_{k}\left(\mathrm{~m}^{2} \mathrm{~K} / \mathrm{W}\right)$ \\
\hline & 4000 & 800 & 0.2 & 10 & $8 \times 10^{-8}$ \\
\hline
\end{tabular}

a $\beta$ : aspect ratio; $\beta_{e}$ : effective aspect ratio; $\delta$ : straightness ratio; $L_{e}$ : average end to end distance; $R_{k}$ : Kaptiza resistance.

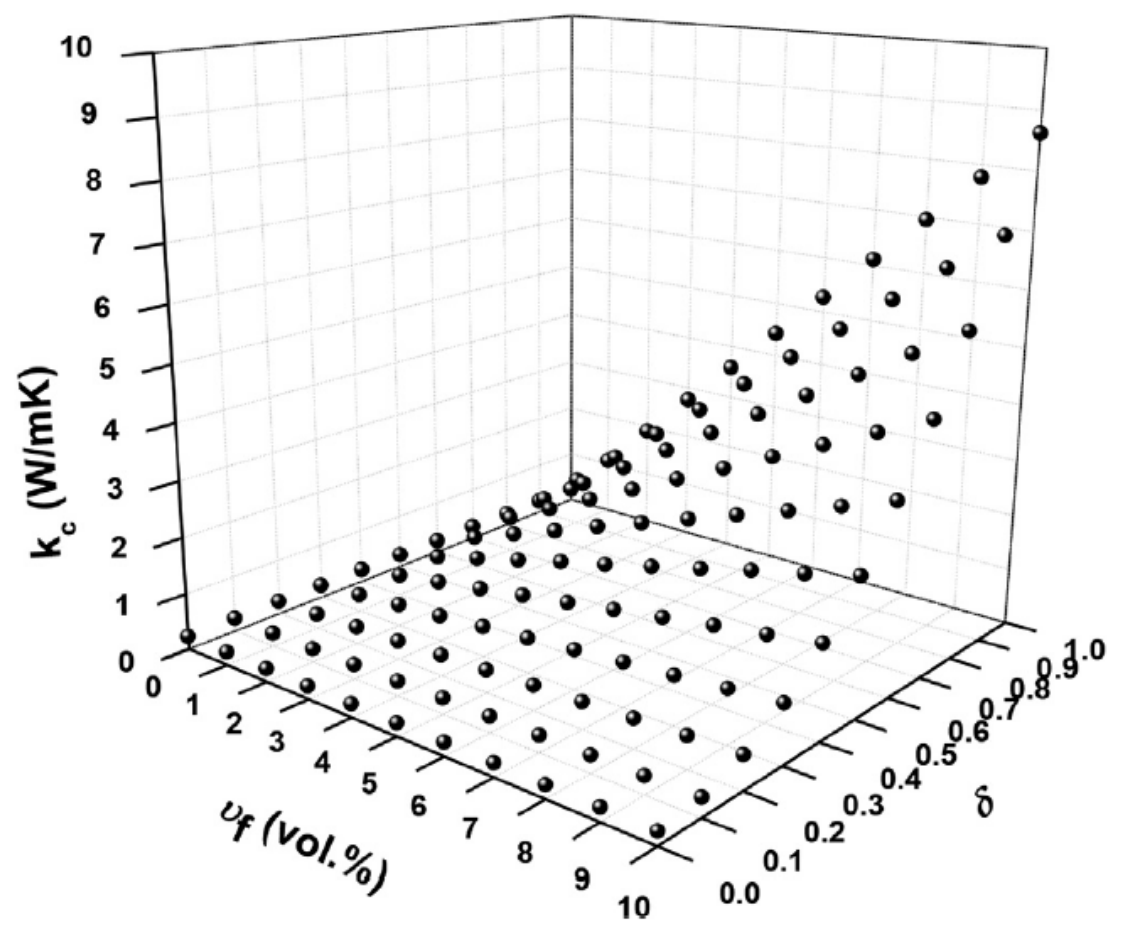

Fig. 7. Prediction of thermal conductivity of PT-30/MWCNTs composites at different fraction and straightness ratio of MWCNTs.

\section{Conclusions}

The incorporations of carbon fillers showed different enhancement effects on the electrical and thermal conductivity of PT-30 resins. MWCNTs, CB and graphite exhibited percolation threshold at $0.1 \mathrm{wt} \%, 0.5 \mathrm{wt} \%$ and $10 \mathrm{wt} \%$, respectively. The maximal electrical conductivity of the composites was $1.08 \mathrm{~S} / \mathrm{cm}, 9.94$ 10 $3 \mathrm{~S} / \mathrm{cm}$ and $1.70 \quad 10$ $5 \mathrm{~S} / \mathrm{cm}$, which was observed at the filler loading of $10 \mathrm{wt} \%$. MWCNTs showed the best enhancement on the electrical conductivity. In contrast, graphite can hardly increase the electrical conductivity. According to the SEM observations, the inter-particle gaps were substantial that prevented the tunnelling of electrons. The percolated network was not formed at low loadings. On the other hand, incorporation of MWCNTs, CB and graphite increased the thermal conductivity of PT-30 resin by $90 \%, 15 \%$ and $92 \%$. Theoretic models were introduced to correlate the experimental results of PT-30/MWCNTs composites. The interfacial thermal resistance of MWCNTs was $810-8 \mathrm{~m} 2 \mathrm{~K} / \mathrm{W}$ and the straightness ratio was 0.2 . It indicates the unexplored potential of MWCNTs for a further enhancement. 


\section{Acknowledgement}

This work was supported by the National Natural Science Foundation of China [grant number 51503205]; and the Chinese Academy of Sciences [2015.90] "Light of West China" Program.

\section{References}

[1] H.S. Kim, J. Jang, J. Yu, S.Y. Kim, Thermal conductivity of polymer composites based on the length of multi-walled carbon nanotubes, Compos. Part B Eng. 79 (2015) 505-512.

[2] J. Huang, Y. Zhu, L. Xu, J. Chen, W. Jiang, X. Nie, Massive enhancement in the thermal conductivity of polymer composites by trapping graphene at the interface of a polymer blend, Compos. Sci. Technol. 129 (2016) 160-165.

[3] H. Chen, V.V. Ginzburg, J. Yang, Y. Yang, W. Liu, Y. Huang, et al., Thermal conductivity of polymer-based composites: fundamentals and applications, Prog. Polym. Sci. 59 (2016) 41-85.

[4] I. Burmistrov, N. Gorshkov, I. Ilinykh, D. Muratov, E. Kolesnikov, S. Anshin, et al., Improvement of carbon black based polymer composite electrical conductivity with additions of MWCNT, Compos. Sci. Technol. 129 (2016) 79-85.

[5] J. Yu, H.K. Choi, H.S. Kim, S.Y. Kim, Synergistic effect of hybrid graphene nanoplatelet and multi-walled carbon nanotube fillers on the thermal conductivity of polymer composites and theoretical modeling of the synergistic effect, Compos. Part A Appl. Sci. Manuf. 88 (2016) 79-85. 
[6] M. Castellino, M. Rovere, M.I. Shahzad, A. Tagliaferro, Conductivity in carbon nanotube polymer composites: a comparison between model and experiment, Compos. Part A Appl. Sci. Manuf. 87 (2016) 237-242.

[7] S.Y. Yang, W.N. Lin, Y.L. Huang, H.W. Tien, J.Y. Wang, C.C.M. Ma, et al., Synergetic effects of graphene platelets and carbon nanotubes on the mechanical and thermal properties of epoxy composites, Carbon 49 (2011) 793-803.

[8] F.H. Gojny, M.H.G. Wichmann, B. Fiedler, I.A. Kinloch, W. Bauhofer, A.H. Windle, et al., Evaluation and identification of electrical and thermal conduction mechanisms in carbon nanotube/epoxy composites, Polymer 47 (2006) 2036-2045.

[9] H. Kim, A.A. Abdala, C.W. MacOsko, Graphene/polymer nanocomposites, Macromolecules 43 (2010) 6515-6530.

[10] X. Sun, J. Jin, X. Wang, D. Cai, M. Song, Conductive behaviour and selfconductance characteristic of carbon nanotubes/functionalized graphene hybrid films, J. Nanosci. Nanotechnol. 11 (2011) 5075-5082.

[11] J. Yang, J. Liang, A modified model of electrical conduction for carbon blackpolymer composites, Polym. Int. 60 (2011) 738-742.

[12] Q. Zhang, J. Wu, L Gao, T. Liu, W. Zhong, G. Sui, et al., Dispersion stability of functionalized MWCNT in the epoxy-amine system and its effects on mechanical and interfacial properties of carbon fiber composites, Mat. Des. 94 (2016) 392-402.

[13] A. Patti, P. Russo, D. Acierno, S. Acierno, The effect of filler functionalization on dispersion and thermal conductivity of polypropylene/multi wall carbon nanotubes composites, Compos. Part B Eng. 94 (2016) 350-359.

[14] Y.J. Noh, S.Y. Pak, S.H. Hwang, J.Y. Hwang, S.Y. Kim, J.R. Youn, Enhanced dispersion for electrical percolation behavior of multi-walled carbon nanotubes in polymer nanocomposites using simple powder mixing and in situ polymerization with surface treatment of the fillers, Compos. Sci. Technol. 89 (2013) 29-37.

[15] A. Allaoui, S. Bai, H. Cheng, J. Bai, Mechanical and electrical properties of a MWNT/epoxy composite, Compos. Sci. Technol. 62 (2002) 1993-1998.

[16] J. Yan, T. Wei, Z. Fan, W. Qian, M. Zhang, X. Shen, et al., Preparation of graphene nanosheet/carbon nanotube/polyaniline composite as electrode material for supercapacitors, J. Power Sources 195 (2010) 3041-3045.

[17] J. Jin, Y. Lin, M. Song, C. Gui, S. Leesirisan, Enhancing the electrical conductivity of polymer composites, Eur. Polym. J. 49 (2013) 1066-1072.

[18] S.H. Goodman, H. Dodiuk-Kenig, Handbook of Thermoset Plastics, third ed., William Andrew, 2014.

[19] J.-E. An, Y.G. Jeong, Structure and electric heating performance of graphene/ epoxy composite films, Eur. Polym. J. 49 (2013) 1322-1330.

[20] F. El-Tantawy, K. Kamada, H. Ohnabe, In situ network structure, electrical and thermal properties of conductive epoxy resin-carbon black composites for electrical heater applications, Mat. Lett. 56 (2002) 112-126.

[21] J.H. Lee, Y.K. Jang, C.E. Hong, N.H. Kim, P. Li, H.K. Lee, Effect of carbon fillers on properties of polymer composite bipolar plates of fuel cells, J. Power Sources 193 (2009) 523-529.

[22] F. Vahedi, H.R. Shahverdi, M.M. Shokrieh, M. Esmkhani, Effects of carbon 
nanotube content on the mechanical and electrical properties of epoxy-based composites, New Carbon Mater 29 (2014) 419-425.

[23] M.-T. Hung, O. Choi, Y.S. Ju, H.T. Hahn, Heat conduction in graphitenanoplatelet-reinforced polymer nanocomposites, Appl. Phys. Lett. 89 (2006) 023117.

[24] S.-Y. Yang, W.-N. Lin, Y.-L. Huang, H.-W. Tien, J.-Y. Wang, C.-C.M. Ma, et al., Synergetic effects of graphene platelets and carbon nanotubes on the mechanical and thermal properties of epoxy composites, Carbon 49 (2011) 793-803.

[25] S. Gantayat, G. Prusty, D.R. Rout, S.K. Swain, Expanded graphite as a filler for epoxy matrix composites to improve their thermal, mechanical and electrical properties, New Carbon Mater 30 (2015) 432-437.

[26] Q. Lin, L. Qu, Q. Lü, C. Fang, Preparation and properties of graphene oxide nanosheets/cyanate ester resin composites, Polym. Test. 32 (2013) 330-337.

[27] X. Wang, J. Jin, M. Song, Cyanate ester resin/graphene nanocomposite: curing dynamics and network formation, Eur. Polym. J. 48 (2012) 1034-1041.

[28] A. Toldy, Á. Szlancsik, B. Szolnoki, Reactive flame retardancy of cyanate ester/ epoxy resin blends and their carbon fibre reinforced composites, Polym. Degrad. Stab. 128 (2016) 29-38.

[29] X. Zhan, L. Wang, J. Zhang, J. Cheng, Synthesis, properties and cure chemistry of a novel room-temperature liquid cyanate ester, Mat. Des. 88 (2015) $1100-1108$.

[30] L. Baikova, T. Pesina, D. Sakseyev, A. Fainleib, V. Bershtein, Hybrid cyanate ester resin-based nanocomposites: increased indentation size effect due to anomalous composition of micron subsurface layer, Polym. Test. 53 (2016) $15-18$.

[31] T.J. Wooster, S. Abrol, J.M. Hey, D.R. MacFarlane, Thermal, mechanical, and conductivity properties of cyanate ester composites, Compos. Part A Appl. Sci. Manuf. 35 (2004) 75-82.

[32] C. Han, A. Gu, G. Liang, L. Yuan, Carbon nanotubes/cyanate ester composites with low percolation threshold, high dielectric constant and outstanding thermal property, Compos. Part A Appl. Sci. Manuf. 41 (2010) 1321-1328.

[33] B. Wang, Y. Jiao, A. Gu, G. Liang, L. Yuan, Dielectric properties and mechanism of composites by superposing expanded graphite/cyanate ester layer with carbon nanotube/cyanate ester layer, Compos. Sci. Technol. 91 (2014) 8-15.

[34] Y. Lin, C.A. Stone, S.J. Shaw, M. Song, Effect of carbon nanotubes on the curing dynamics and network formation of cyanate ester resin, J. Polym. Res. 20 (2013) 106.

[35] C.P. Camirand, Measurement of thermal conductivity by differential scanning calorimetry, Thermochim. Acta 417 (2004) 1-4.

[36] F. Deng, Q.-S. Zheng, L-F. Wang, C.-W. Nan, Effects of anisotropy, aspect ratio, and nonstraightness of carbon nanotubes on thermal conductivity of carbon nanotube composites, Appl. Phys. Lett. 90 (2007) 021914.

[37] S.T. Huxtable, D.G. Cahill, S. Shenogin, L Xue, R. Ozisik, P. Barone, et al., Interfacial heat flow in carbon nanotube suspensions, Nat. Mater 2 (2003) 731-734. 